\title{
Effect of obesity on the prognosis and recurrence of prostate cancer after radical prostatectomy: a meta-analysis
}

\author{
Runtian Luo, Yongbo Chen, Ke Ran, Qing Jiang \\ Department of Urology, the Second Affiliated Hospital of Chongqing Medical University, Chongqing, China \\ Contributions: (I) Conception and design: R Luo, Q Jiang; (II) Administrative support: Q Jiang; (III) Provision of study materials or patients: Y Chen, \\ K Ran; (IV) Collection and assembly of data: R Luo, Y Chen, K Ran; (V) Data analysis and interpretation: R Luo, Q Jiang; (VI) Manuscript writing: \\ All authors; (VII) Final approval of manuscript: All authors. \\ Correspondence to: Qing Jiang. Department of Urology, the Second Affiliated Hospital of Chongqing Medical University, Chongqing, China. \\ Email: 745495489@qq.com.
}

\begin{abstract}
Background: Obesity has been found to be closely related to the increased risk of fatal prostate cancer (PCa), however there remains no evidence that further clarifies the relationship between obesity and the postoperative recurrence and poor prognosis of PCa. In this study, a systematic review and meta-analysis were performed to systematically evaluate the effect of obesity on the prognosis and recurrence of PCa after radical prostatectomy $(\mathrm{RP})$.
\end{abstract}

Methods: A literature search of the PubMed, Web of Science, and Embase databases was performed covering articles published between January 2013 and January 2020. Articles regarding the correlation between body mass index (BMI) and the prognosis and recurrence of PCa following RP were included in the meta-analysis. Two investigators independently screened the literature and extracted relevant data including publication information, key results, number of cancer cases, and multivariable-adjusted odds ratios (ORs) with $95 \%$ confidence intervals (CIs). Meta-analysis was performed using RevMan 5.3 and Stata 16.0 software, and forest plots, funnel plots, and sensitivity analysis were also conducted.

Results: A total of 14 articles were included, all of which were analyzed for clinicopathological characteristics. Eight articles reported the biochemical recurrence (BCR) with prostate-specific antigen (PSA) as the predictor, and six articles reported the positive surgical margins (PSM). The meta-analysis showed that obese PCa patients had more postoperative recurrence and poor prognosis compared with the normal weight PCa patients, and the difference was statistically significant (OR =1.25, 95\% CI: 1.10, 1.43). BCR exhibited no significant difference between obese and non-obese PCa patients after surgery (OR =1.2, 95\% CI: 0.96, 1.46), and there were also no notable differences in PSM between the groups (OR =1.16, 95\% CI: 0.99, 1.36). Subgroup analysis showed that obese PCa patients in the Americas (95\% CI: 1.11, 1.37) and Europe (95\% CI: $1.11,1.78)$ were more likely to have surgical recurrence and poor prognosis $(\mathrm{OR}=1.40)$. Obese patients in the Americas were also more likely to have BCR after surgery (95\% CI: 1.07, 1.36).

Conclusions: Obesity easily leads to poor prognosis and recurrence of PCa after RP.

Keywords: Obesity; prostate cancer (PCa); prognosis; recurrence; meta-analysis

Submitted Sep 15, 2020. Accepted for publication Dec 01, 2020.

doi: $10.21037 /$ tau-20-1352

View this article at: http://dx.doi.org/10.21037/tau-20-1352

\section{Introduction}

Prostate cancer $(\mathrm{PCa})$ is a common malignancy that poses a threat to the health of men. It has been reported that in 185 countries, 1.3 million new cases and 360,000 deaths were caused by PCa in 2018, making it the second most prevalent disease among men (1). In China, PCa ranked the sixth among all male cancers in 2015 and its incidence has exhibited a significant upward trend (2). Owing to 
the insidious onset of $\mathrm{PCa}$, patients are typically already in advanced stages at the time of diagnosis, resulting in poor prognosis and high mortality (3). Therefore, regular postoperative detection of patients is key to preventing poor prognosis and recurrence of PCa. At present, endocrine therapy, radical prostatectomy (RP) and external radiotherapy are applied in the clinical treatment of PCa. At the same time, gene therapy has gradually attracted people's attention. There are many methods to treat PCA by modifying gene and gene immune regulation (4). In clinical diagnosis, biochemical recurrence (BCR) with serum prostate-specific antigen (PSA) as the predictor and positive surgical margins (PSM) are used to evaluate PCa recurrence and prognosis after RP and external radiotherapy (5).

The risk factors of prostate cancer include age, heredity, region, race and gene change, among which age is the biggest risk factor. On the other hand, obesity is related to diabetes, hypertension, atherosclerosis and other diseases. In recent studies, obesity has been found to be closely related to the increased risk of fatal $\mathrm{PCa}$ (6), which accelerates the development of PCa and increases mortality both before and after diagnosis. Ma et al. found that a one-unit increase of BMI in obese people led to an approximately $10 \%$ increase in the risk of $\mathrm{PCa}$ compared with normal weight individuals, and obese people with $\mathrm{BMI} \geq 30 \mathrm{~kg} / \mathrm{m}^{2}$ led to a $20 \%$ increase in the risk of PCa (7). In the metaanalysis conducted by Cao et al., a $5 \mathrm{~kg} / \mathrm{m}^{2}$ increase of $\mathrm{BMI}$ was found to increase the risk of PCa-specific death by $20 \%$ (8). These findings indicate that obesity is closely associated with the development of PCa. Furthermore, the mortality rate of obese colorectal cancer patients is more than three times higher than that of normal weight patients (9). In addition, obesity also leads to recurrence and poor prognosis in breast and lung cancers $(10,11)$. Thus, it is clear that obesity is a risk factor for postoperative recurrence and poor prognosis in most cancers.

At present, there are numerous studies addressing the relationship between obesity and the occurrence of $\mathrm{PCa}$, however there remains no evidence that further clarifies the relationship between obesity and the postoperative recurrence and poor prognosis of PCa. Although there are many studies on the incidence rate of obesity and its incidence, this study is the first time to perform a systematic review and meta-analysis to evaluate the relationship between obesity and the prognosis of RP and PCa recurrence, so as to provide a reference for the clinical treatment and prognosis evaluation of obese patients with PCa.
We present the following article in accordance with the PRISMA reporting checklist (available at http://dx.doi. org/10.21037/tau-20-1352).

\section{Methods}

\section{Literature retrieval strategy}

A literature search of the PubMed, Web of Science, and Embase databases was performed in English for relevant articles published between January 2013 and January 2020. The search terms were as follows: ("obesity" or "BMI" or "body mass index" or "overweight" or "adiposity") and ("prostate cancer" or "prostatectomy" or "radical prostatectomy"). The references of retrieved literature were also tracked to find relevant papers.

\section{Screening criteria}

Inclusion criteria were as follows: (I) case-control studies on obesity and the prognosis and recurrence of $\mathrm{PCa}$ after RP with human study subjects; (II) complete original data, or enough data to calculate the odds ratio (OR) with $95 \%$ confidence interval (CI).

Exclusion criteria were as follows: (I) comments, reviews, meta-analyses, animal studies, or in vitro cell studies; (II) articles irrelevant to the topic, repetitive studies, and studies with insufficient data and design flaws; (III) studies with unclear conclusions.

\section{Study indicators}

\section{Criteria of obesity}

The data collected in this study were divided into the test group (obesity population) and the control group (normal weight population). If there were multiple groups in the original literature, the population with body weight $>30 \mathrm{~kg} / \mathrm{m}^{2}$ was classified as the test group.

\section{Related indicators to evaluate surgical prognosis and recurrence}

The serum PSA level was measured every 3 months; PSA $>0.2 \mathrm{ng} / \mathrm{mL}$ in two successive tests after surgery was considered as BCR. PSM is a common indicator in adjuvant radiotherapy and measures the quality of surgery; so the surgical prognosis was primarily evaluated by the PSM of RP specimens. 


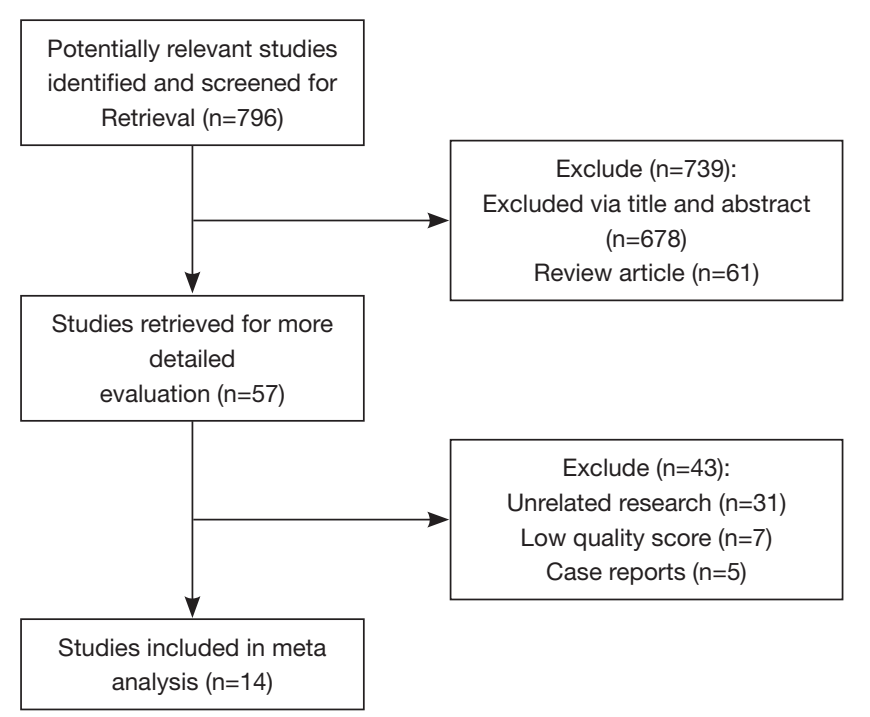

Figure 1 Literature screening process.

\section{Data extraction}

Two investigators independently screened the literature and extracted the data. The literature screening process was as follows: first, studies that did not meet the screening criteria requirements were excluded. Next, studies were excluded by reading the abstracts or the full text if necessary. Finally, the corresponding materials were cross-checked to determine the studies included in this meta-analysis.

The following data were extracted: first author's name, year of publication, country, study design, main reported outcomes, number of cancer cases, total number of subjects, study duration, adjusted confounders, and multivariableadjusted OR with $95 \%$ CI. During the data extraction process, disagreements between the two investigators were resolved by referring to the original literature.

\section{Statistical analysis}

Review manager (RevMan) 5.3 and Stata 16.0 were used for statistical analysis. Dichotomous variables were compared with weighted mean difference (WMD) and OR with 95\% CI. Heterogeneity among the included studies was evaluated using $\mathrm{I}^{2}$ statistics. An $\mathrm{I}^{2}>50 \%$ indicated significant heterogeneity, and in these cases, a random effects model (REM) was used. If the heterogeneity was not significant, a fixed effects model (FEM) was applied. Sensitivity analysis and publication bias were analyzed using Stata 16.0 (StataCorp LLC, college station, TX, USA).

\section{Results}

\section{Basic characteristics of the included literature}

A total of 796 potentially relevant studies were retrieved according to the retrieval strategy. Of these, 678 irrelevant studies were excluded through reading titles and abstracts, and a further 61 review articles were also excluded. The remaining 57 articles were included. After reading the full texts, 31 unrelated articles, seven studies with a low quality score, and five case reports were also excluded. Finally, 14 articles were included in this meta-analysis. The specific literature screening process and results are shown in Figure 1, and the basic characteristics of the 14 included articles are displayed in Table 1.

\section{Comparison of postoperative recurrence and prognosis in obese and non-obese PCa patients}

A total of 14 articles regarding obesity and postoperative recurrence and prognosis were included (12-25). As shown in Figure 2, a significant heterogeneity among the studies was identified $\left(\mathrm{I}^{2}=71.0 \%, \mathrm{P}=0.000\right)$, so the $\mathrm{REM}$ was used. It was confirmed that obese PCa patients had more postoperative recurrence and poor prognosis compared with the non-obese PCa patients ( $\mathrm{OR}=1.25,95 \% \mathrm{CI}: 1.10,1.43$, $\mathrm{P}<0.001)$. As $\mathrm{I}^{2}>50 \%$, which indicated high heterogeneity among the studies, a subgroup analysis was carried out to determine the source of heterogeneity. It was divided into three groups according to region: Asia $(\mathrm{OR}=1.22,95 \% \mathrm{CI}$ : $0.86,1.74)$, the Americas (OR $=1.23,95 \%$ CI: $1.11,1.37$ ), and Europe (OR $=1.40,95 \%$ CI: 1.11, 1.78) (Figure 2). The results showed no heterogeneity among different regions. Also, the funnel plot was symmetrical, indicating a lower probability of publication bias in the included literature (Figure 3). Sensitivity analysis was performed by removing the studies one by one; the result showed that the pooled effect size was still statistically significant, and the forest plot direction did not change significantly before and after removal (Figure 4).

\section{BCR conditions in obese and non-obese PCa patients after surgery}

A total of eight articles were included to report the BCR condition of patients $(15,18-20,22-25)$. Firstly, obvious heterogeneity among the studies was identified $\left(\mathrm{I}^{2}=79.2 \%\right.$, $\mathrm{P}<0.001$ ) (Figure $5 A$ ), so the REM was used for meta- 
Table 1 Basic characteristics of the included literature

\begin{tabular}{|c|c|c|c|c|c|c|c|c|c|}
\hline No. & First author & Year & Sample time & Country & $\begin{array}{l}\text { Sample } \\
\text { size }\end{array}$ & Age (years) & $\begin{array}{l}\text { Follow-up time } \\
\text { (months) }\end{array}$ & Study design & $\begin{array}{l}\text { Outcome } \\
\text { measured }\end{array}$ \\
\hline 1 & Lijiang Sun & 2019 & 2005-2014 & China & 422 & 68 [47-82] & 19.4 [0.3-88.9] & Retrospective & PSM \\
\hline 2 & Wen Liu & 2020 & 2005-2014 & China & 1,016 & 68 [41-79] & NR & Retrospective & PSM \\
\hline 3 & Farnoosh Nik-Ahd & 2020 & $1988-2017$ & US & 4,766 & 63 [58-66] & 88.8 & Retrospective & PSM \\
\hline 6 & Agnes B. Maj-Hes & 2017 & $2000-2011$ & Austria & 6,519 & $61[57-66]$ & 28 [21-49] & Retrospective & PSM \\
\hline 7 & R Asmar & 2013 & 1994-2007 & US & 1,428 & 59.1 & 43.2 & Retrospective & $\mathrm{BCR}$ \\
\hline 8 & Michel Wissing & 2019 & 2006-2013 & Canada & 1,714 & $41-80$ & 69 [54-87] & Retrospective & BCR \\
\hline 12 & Ilir Agalliu & 2015 & 2005-2012 & US & 610 & 60 & 30.8 & Retrospective & $\mathrm{BCR}$ \\
\hline 13 & Young Dong Yu & 2018 & 2006-2017 & South Korea & 2,997 & $66.0 \pm 6.8$ & 39.6 & Retrospective & $\mathrm{BCR}$ \\
\hline 14 & Heather J. Chalfin & 2014 & 1982-2012 & US & 11,152 & 58 & 60 [12-324] & Retrospective & BCR \\
\hline
\end{tabular}

NR, not reported; BCR, biochemical recurrence; PSM, positive surgical margins; WC, wound complications [including superficial surgical site infection (SSI), deep SSI, organ/space SSI and dehiscence].

analysis. The meta-analysis showed no significant difference in BCR incidence between obese and non-obese patients after surgery ( $\mathrm{OR}=1.20,95 \% \mathrm{CI}: 0.96,1.49)$. Secondly, due to the high heterogeneity, a subgroup analysis was carried out to determine the source of heterogeneity. It was divided according to region into an Asia subgroup and an Americas subgroup. The result of subgroup analysis showed that the heterogeneity was from the literature in Asia; meanwhile, obese patients in the Americas were relatively prone to BCR after surgery ( $\mathrm{OR}=1.21,95 \% \mathrm{CI}: 1.07,1.36)$. To determine the sensitivity of the meta-analysis results, the studies were eliminated one by one; the result showed that the pooled effect size was still statistically significant, and the forest plot direction did not change significantly before and after removal (Figure 5B).

\section{PSM conditions in obese and non-obese PCa patients after surgery}

A total of five articles were included to report the PSM condition of patients $(12-14,17,21)$. Firstly, obvious heterogeneity was identified among the studies $\left(I^{2}=50.9 \%\right.$, $\mathrm{P}<0.001$ ) (Figure $6 A$ ), so the REM was used for meta- analysis. The meta-analysis showed no significant difference in PSM condition between obese and non-obese patients after surgery (OR $=1.16,95 \% \mathrm{CI}: 0.99,1.36)$. Secondly, due to the high heterogeneity, a subgroup analysis was carried out to determine the source of heterogeneity. It was divided according to region into an Asia subgroup, an Americas subgroup, and a Europe subgroup. The result of the subgroup analysis showed that the heterogeneity was from the literature in different regions; meanwhile, obese patients in Asian were relatively prone to PSM after surgery (OR $=1.36,95 \%$ CI: $0.91,2.02$ ). To determine the sensitivity of the meta-analysis results, the studies were eliminated one by one; the result showed that the pooled effect size was still statistically significant, and the forest plot direction did not change significantly before and after removal (Figure 6B).

\section{Discussion}

The tumor microenvironment is crucial in the occurrence, development, and progression of cancers. For example, chronic inflammation is an important factor driving the development of cancer, and epidemiological data shows a close relationship between chronic inflammation and the 


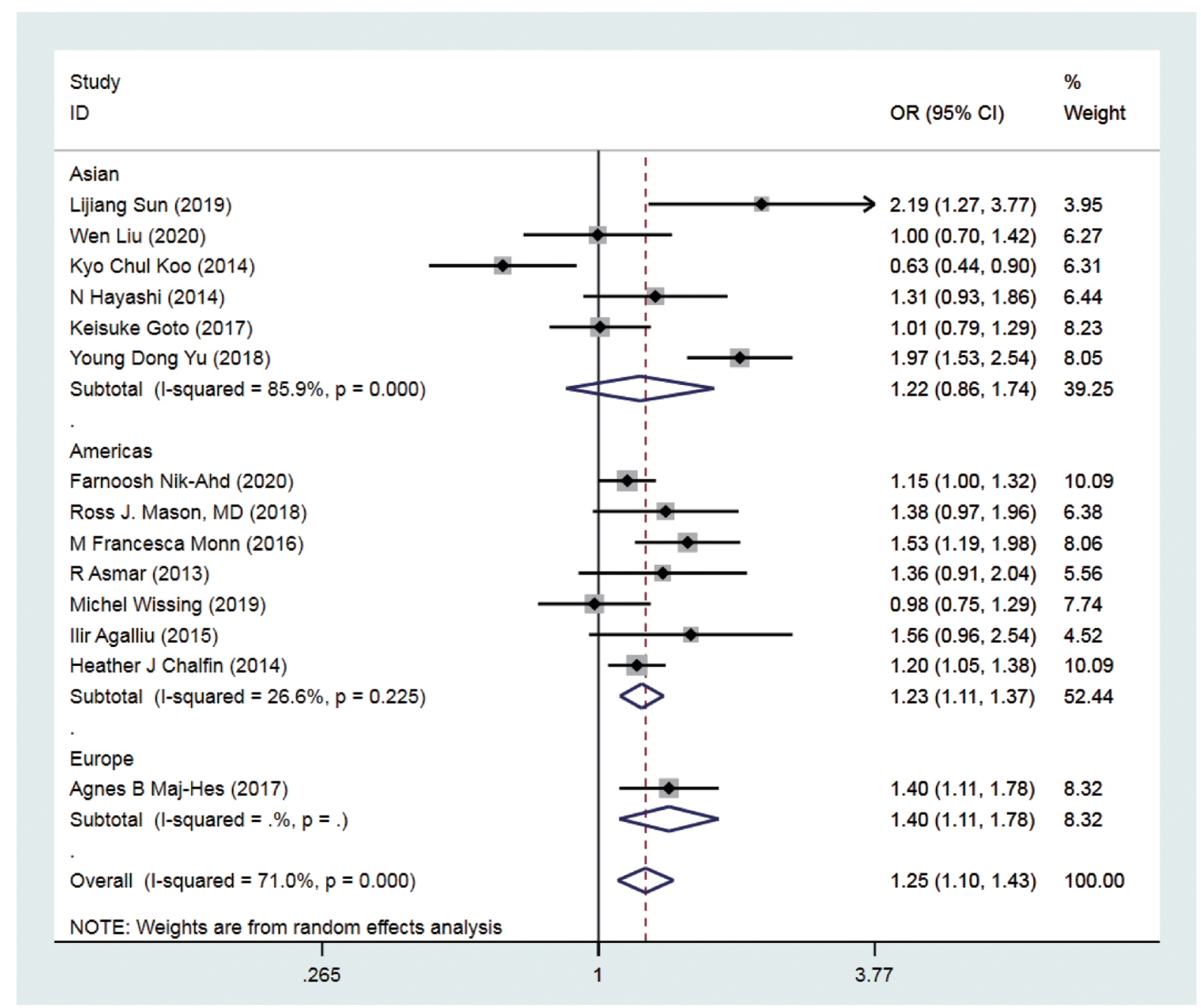

Figure 2 Forest plot of postoperative recurrence and poor prognosis in obese and non-obese PCa patients. OR, odds ratio; CI, confidence interval; PCa, prostate cancer.

progression of liver, pancreatic, and bladder cancers (26). Obesity is usually caused by excessive deposition of adipose tissue, which can easily induce abnormal metabolism of steroid hormones and cytokines, thus resulting in chronic inflammation (27). Studies have found that obesity-related cancers, such as breast cancer and cancers of internal organs, occur near fat accumulation, suggesting that biological changes in fat often locally promote the occurrence and development of a variety of cancers in people with elevated BMI (28). Dickerman et al. demonstrated that both obesity and weight gain in adults are associated with advanced $\mathrm{PCa}$, as well as the high risk of recurrence and survival of PCa (29). Vidal et al. reported that obesity may have a stronger association with $\mathrm{PCa}$ in some patient subgroups (such as black men) (30).

In this study, obese PCa patients had more postoperative recurrence and poor prognosis compared with non-obese patients. Obesity was a risk factor for recurrence and poor prognosis in PCa patients in Asia, Europe, and the Americas. There was significant heterogeneity among the studies caused by differences in patient characteristics, treatment, BMI measurement time, or follow-up time. More studies are required to investigate and determine the relevant reasons.

BCR has been defined as an indicator of PCa recurrence after RP or radiotherapy (31). REM was applied in this meta-analysis, and the results showed no significant difference in the incidence of BCR between obese and non- 
obese patients. However, in the Americas, obese patients had a markedly higher incidence of BCR compared with non-obese patients. This is because the inflammatory response of the body leads to the increase of PSA in the blood (32), and there is a possibility of false positives due to over-diagnosis in the PSA test. Furthermore, BMI classification criteria are different in different countries.

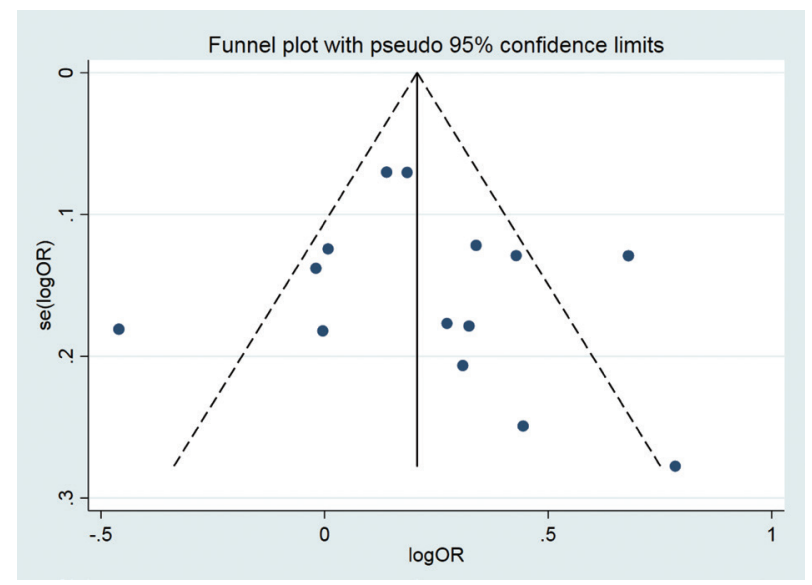

Figure 3 Funnel plot of postoperative recurrence and poor prognosis in obese and non-obese PCa patients. OR, odds ratio; $\mathrm{PCa}$, prostate cancer.
These factors may be responsible for the high incidence of BCR in obese patients in the Americas, resulting in differences in the subgroup analysis.

PSM is a recognized indicator of postoperative quality in $\mathrm{PCa}$, and is also an important indicator of postoperative adjuvant radiotherapy and endocrine therapy (33). As early as 2009, heavier PCa patients were found to have a higher risk of PSM after surgery compared with normal weight individuals (34). In this meta-analysis, REM was used for the combined analysis and the subgroup analysis, and both results revealed no significant difference in the incidence of PSM between obese and non-obese patients. This indicates that with the increasing understanding of $\mathrm{PCa}$ and the development of medical technology, the occurrence of PSM after RP in obese and non-obese patients has been enhanced.

\section{Conclusions}

In summary, this meta-analysis revealed the important relationship between obesity and poor prognosis and recurrence of PCa after RP. However, due to the limited number and quality of the included studies, the above conclusion requires further verification by more highquality studies.

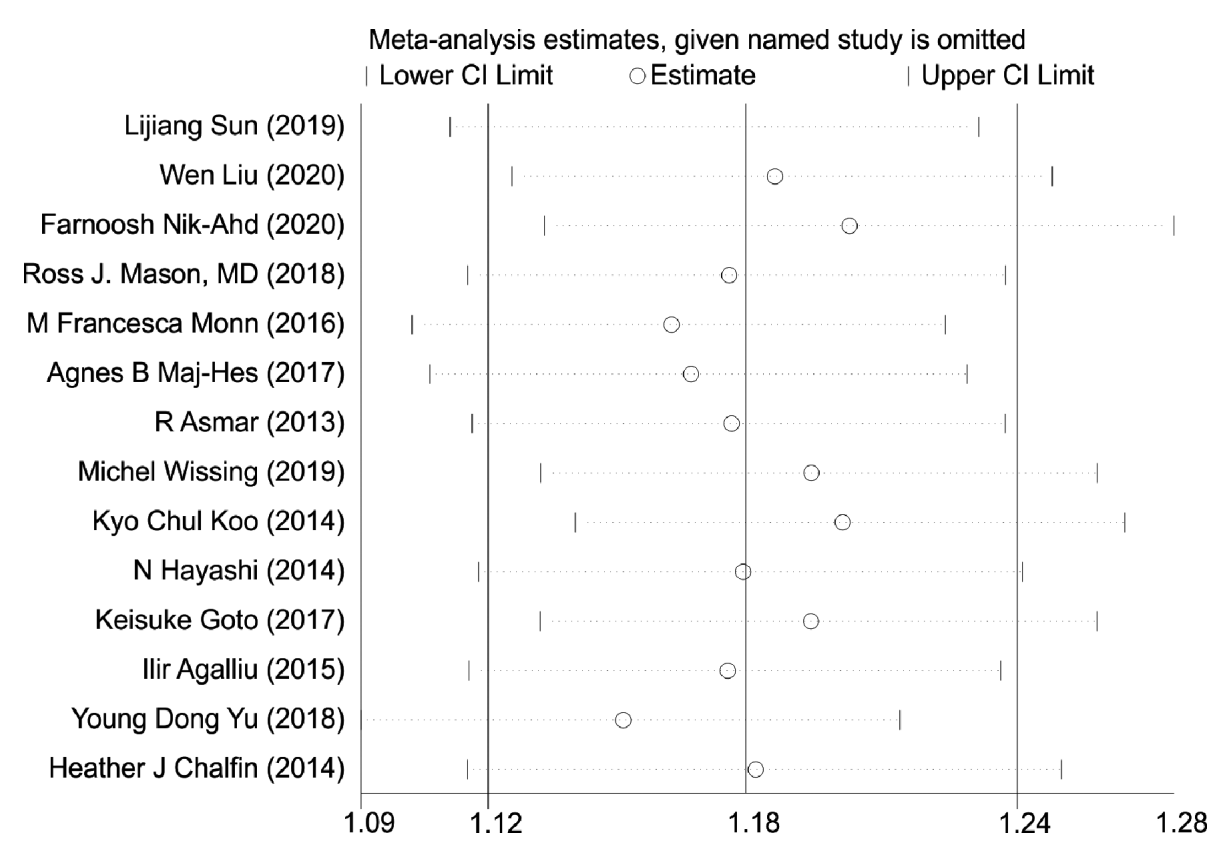

Figure 4 Sensitivity analysis of postoperative recurrence and poor prognosis in obese and non-obese PCa patients. CI, confidence interval; $\mathrm{PCa}$, prostate cancer. 


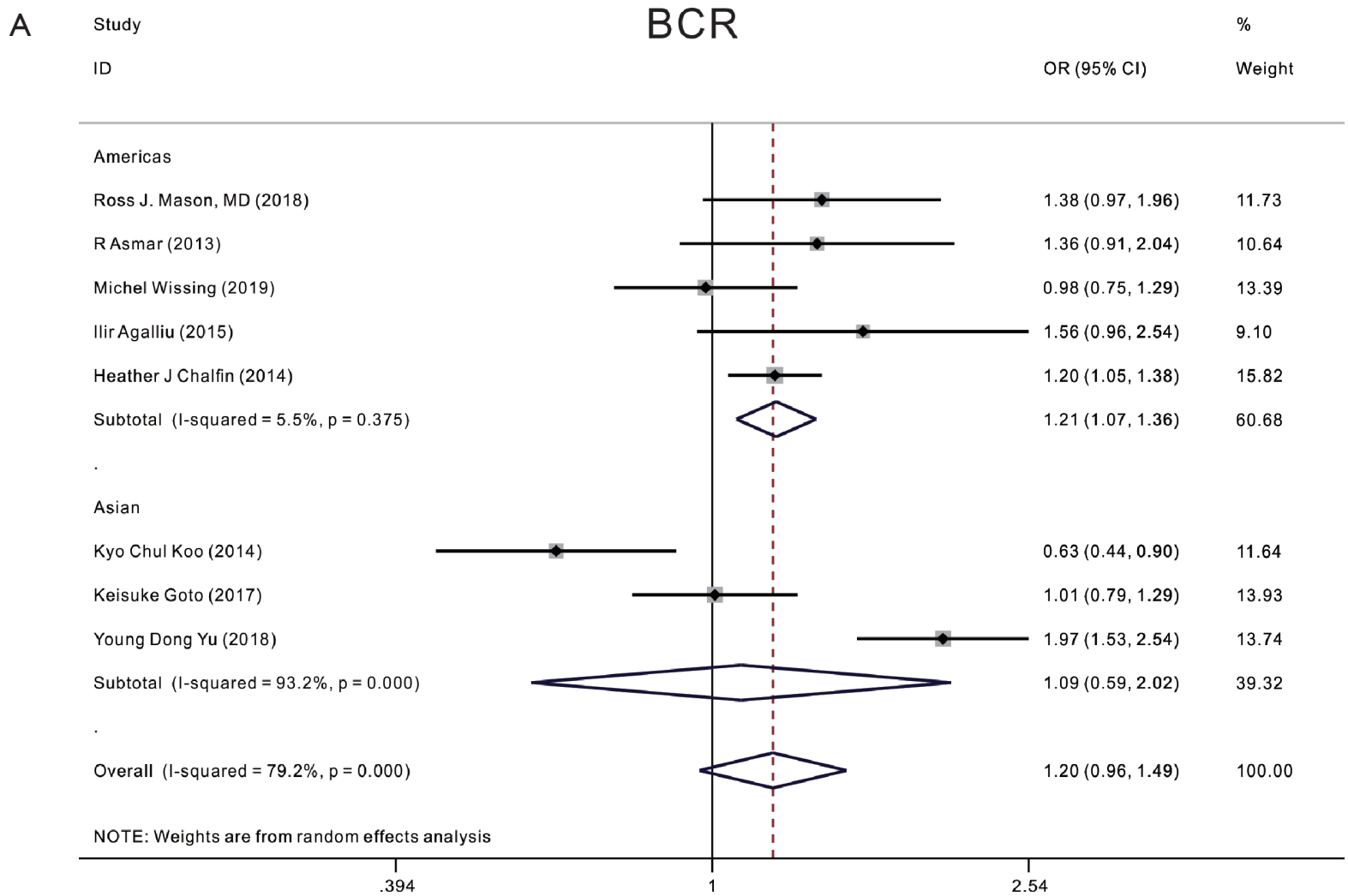

B

Meta-analysis estimates, given named study is omitted

I Lower Cl Limit OEstimate Upper Cl Limit

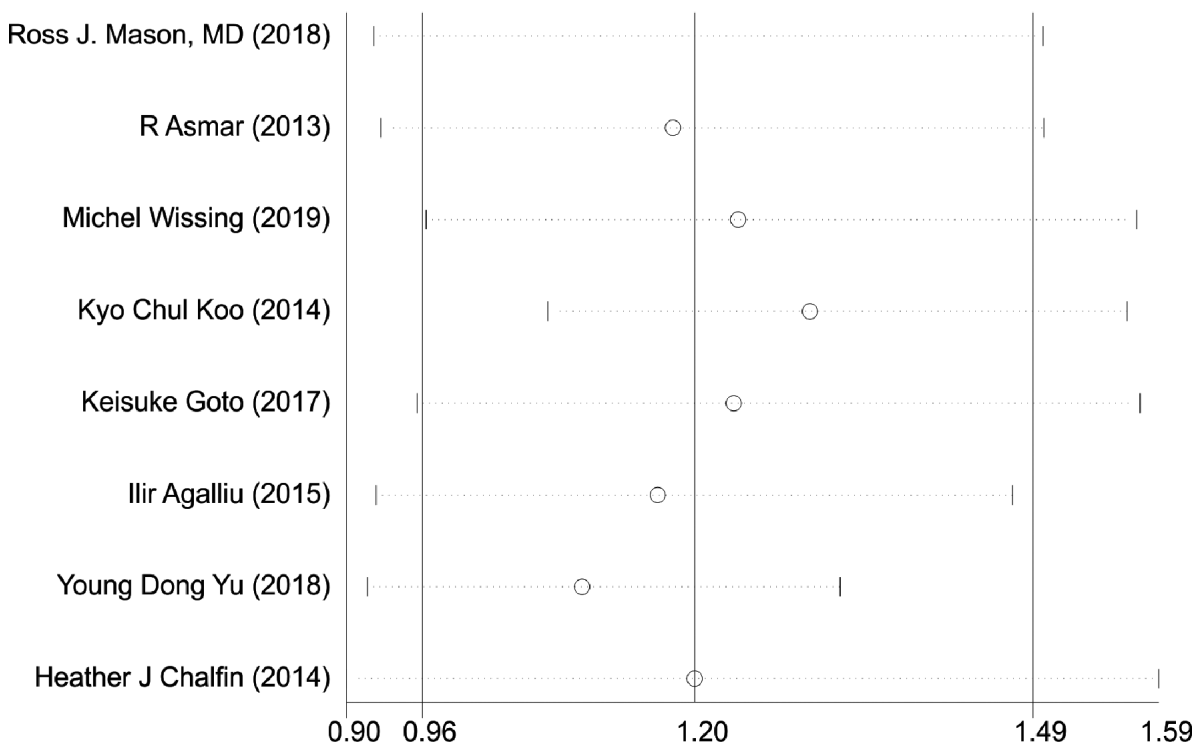

Figure 5 Forest plot (A) and sensitivity analysis plot (B) with BCR as the outcome measure. OR, odds ratio; CI, confidence interval; BCR, biochemical recurrence. 


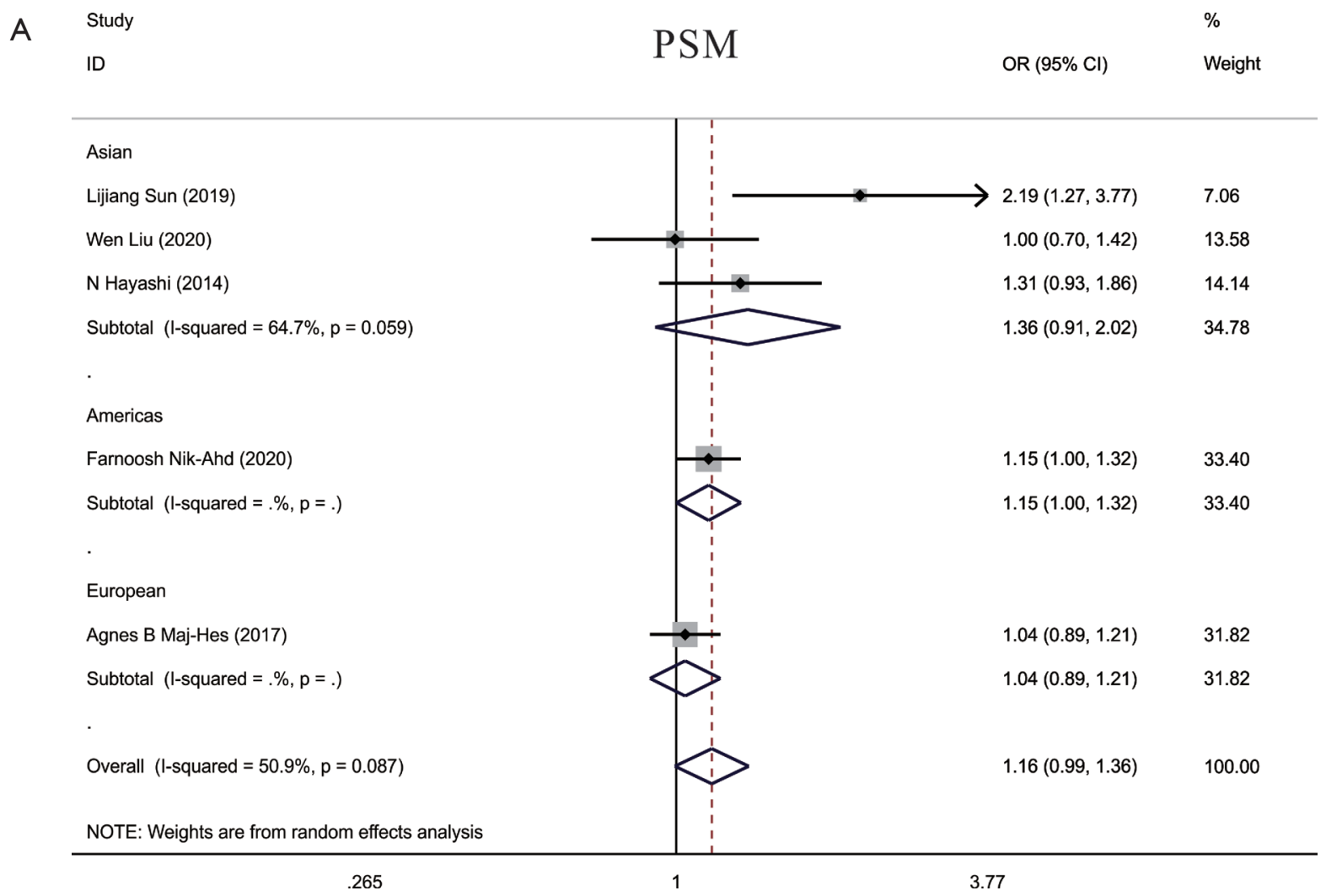

\section{B}

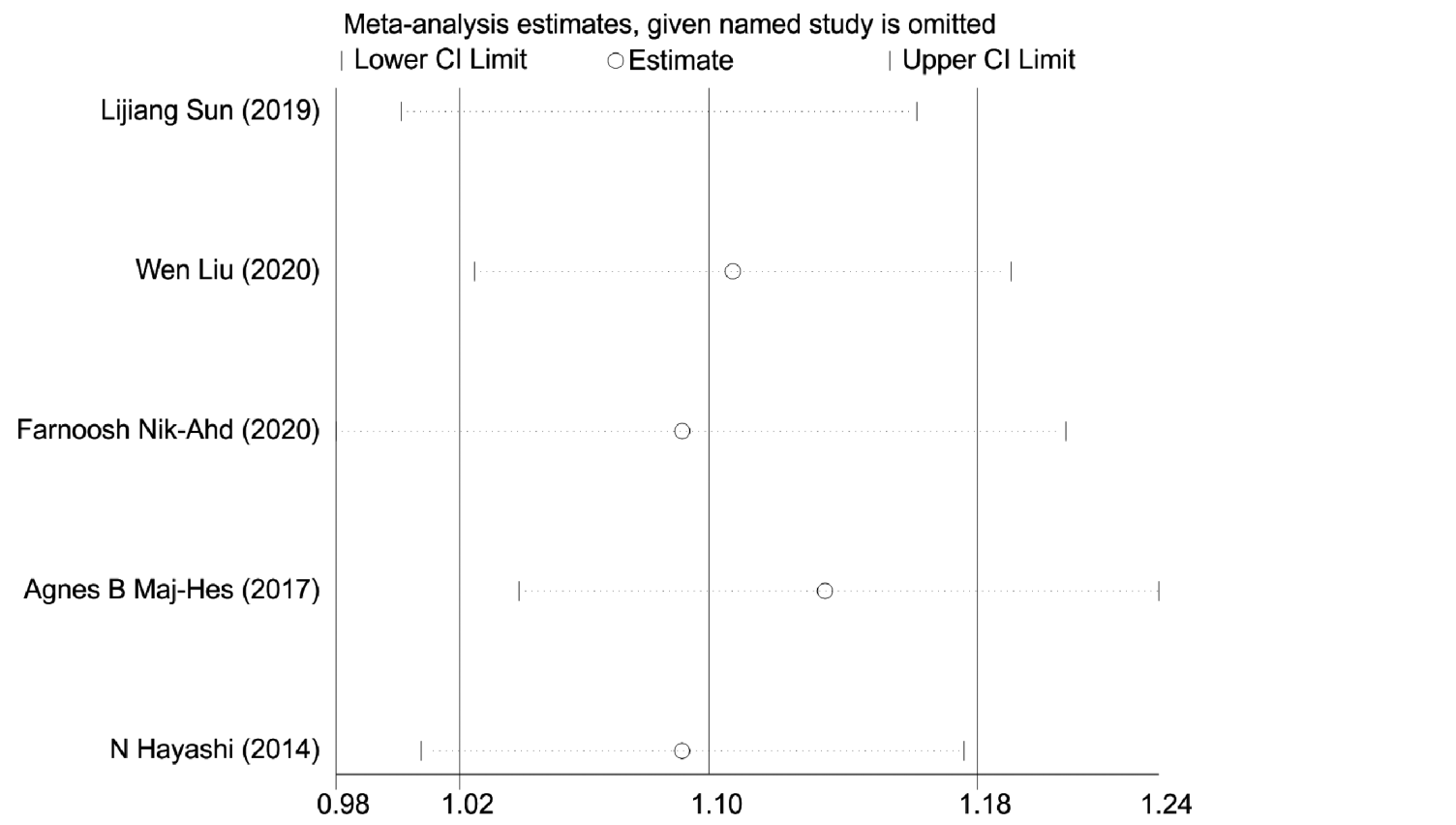

Figure 6 Forest plot (A) and sensitivity analysis plot (B) with PSM as the outcome measure. OR, odds ratio; CI, confidence interval; PSM, positive surgical margin. 


\section{Acknowledgments}

Funding: None.

\section{Footnote}

Reporting Checklist: The authors have completed the PRISMA reporting checklist. Available at http://dx.doi. org/10.21037/tau-20-1352

Conflicts of Interest: All authors have completed the ICMJE uniform disclosure form (available at http://dx.doi. org/10.21037/tau-20-1352). The authors have no conflicts of interest to declare.

Ethical Statement: The authors are accountable for all aspects of the work in ensuring that questions related to the accuracy or integrity of any part of the work are appropriately investigated and resolved.

Open Access Statement: This is an Open Access article distributed in accordance with the Creative Commons Attribution-NonCommercial-NoDerivs 4.0 International License (CC BY-NC-ND 4.0), which permits the noncommercial replication and distribution of the article with the strict proviso that no changes or edits are made and the original work is properly cited (including links to both the formal publication through the relevant DOI and the license). See: https://creativecommons.org/licenses/by-nc-nd/4.0/.

\section{References}

1. Oke J, Fanshawe T. Cancer statistics A survival guide. Significance 2020;17:26-9.

2. Siegel RL, Miller KD, Jemal A. Cancer statistics, 2017. CA Cancer J Clin 2017;67:7-30.

3. Revannasiddaiah S, Gupta I, Devadas SK. Unlocking the potential with the use of check-point inhibitor immunotherapies in metastatic prostate cancer. Chin Clin Oncol 2019;8:S13.

4. Heidenreich A, Bastian PJ, Bellmunt J, et al. EAU Guidelines on Prostate Cancer. Part 1: Screening, Diagnosis, and Local Treatment with Curative IntentUpdate 2013. European Urology 2014;65:124-37.

5. Lim E. Prostate-specific membrane antigen in prostate cancer imaging and treatment. Transl Cancer Res 2018;7:S676-89.

6. Calle EE, Rodriguez C, Walker-Thurmond K, et al.
Overweight, obesity, and mortality from cancer in a prospectively studied cohort of U.S. adults. N Engl J Med 2003;348:1625-38.

7. Ma J, Li H, Giovannucci E, et al. Prediagnostic bodymass index, plasma C-peptide concentration, and prostate cancer-specific mortality in men with prostate cancer: a long-term survival analysis. Lancet Oncol 2008;9:1039-47.

8. Cao Y, Ma J. Body Mass Index, Prostate Cancer-Specific Mortality, and Biochemical Recurrence: a Systematic Review and Meta-analysis. Cancer Prev Res (Phila) 2011;4:486-501.

9. Tao W, Konings P, Hull MA, et al. Colorectal cancer prognosis following obesity surgery in a population-based cohort study. Obes Surg 2017;27:1233-9.

10. Jiralerspong S, Goodwin PJ. Obesity and breast cancer prognosis: evidence, challenges, and opportunities. J Clin Oncol 2016;34:4203-16.

11. Zhang X, Liu Y, Shao H, et al. Obesity paradox in lung cancer prognosis: evolving biological insights and clinical implications. J Thorac Oncol 2017;12:1478-88.

12. Sun L, Xu T, Yuan X, et al. Obesity is a predictor in prostate cancer patients receiving prostatectomy after neoadjuvant hormonal therapy. Tumori 2020;106:133-8.

13. Liu W, Li W, Wang Z, et al. Metabolically Abnormal Obesity Increases the Risk of Advanced Prostate Cancer in Chinese Patients Undergoing Radical Prostatectomy . Cancer Manag Res 2020;12:1779-87.

14. Nik-Ahd F, Howard LE, Aronson WJ, et al. Obese men undergoing radical prostatectomy: Is robotic or retropubic better to limit positive surgical margins? Results from SEARCH. Int J Urol 2020;27:851-7.

15. Mason RJ, Boorjian SA, Bhindi B, et al. Examining the association between adiposity and biochemical recurrence after radical prostatectomy. Can Urol Assoc J 2018;12:E331-E337.

16. Monn MF, Jaqua KR, Calaway AC, et al. Impact of Obesity on Wound Complications Following Radical Prostatectomy Is Mitigated by Robotic Technique. J Endourol 2016;30:890-5.

17. Maj-Hes AB, Mathieu R, Özsoy M, et al. Obesity is associated with biochemical recurrence after radical prostatectomy: A multi-institutional extended validation study. Urol Oncol 2017;35:460.e1-8.

18. Asmar R, Beebe-Dimmer JL, Korgavkar K, et al. obesity and prostate cancer biochemical recurrence after radical prostatectomy. Prostate Cancer Prostatic Dis 2013;16:62-6.

19. Wissing M, Chevalier S, McKercher G, et al. The 
relationship between body-mass index, physical activity, and pathologic and clinical outcomes after radical prostatectomy for prostate cancer. World J Urol 2019;37:789-98.

20. Koo KC, Yoon YE, Rha KH, et al. Low body mass index is associated with adverse oncological outcomes following radical prostatectomy in Korean prostate cancer patients. Int Urol Nephrol 2014;46:1935-40.

21. Hayashi N, Matsushima M, Kido M, et al. BMI is associated with larger index tumors and worse outcome after radical prostatectomy. Prostate Cancer Prostatic Dis 2014;17:233-7.

22. Goto K, Nagamatsu H, Teishima J, et al. Body mass index as a classifier to predict biochemical recurrence after radical prostatectomy in patients with lower prostatespecific antigen levels. Mol Clin Oncol 2017;6:748-752.

23. Agalliu I, Williams S, Adler B, et al. The impact of obesity on prostate cancer recurrence observed after exclusion of diabetics. Cancer Causes Control 2015;26:821-30.

24. Yu YD, Byun SS, Lee SE, et al. Body Mass Index on Oncological Outcomes of Prostate Cancer Patients after Radical Prostatectomy. Sci Rep 2018;8:11962.

25. Chalfin HJ, Lee SB, Jeong BC, et al. Obesity and long-term survival after radical prostatectomy. J Urol 2014;192:1100-4.

26. Mantovani A, Allavena P, Sica A, et al. Cancer-related inflammation. Nature 2008;454:436-44.

27. Rosen ED, Spiegelman BM. What We Talk About When We Talk About Fat. Cell 2014;156:20-44.

Cite this article as: Luo R, Chen Y, Ran K, Jiang Q. Effect of obesity on the prognosis and recurrence of prostate cancer after radical prostatectomy: a meta-analysis. Transl Androl Urol 2020;9(6):2713-2722. doi: 10.21037/tau-20-1352
28. van Kruijsdijk RC, van der Wall E, Visseren FL. Obesity and cancer: the role of dysfunctional adipose tissue. Cancer Epidemiol Biomarkers Prev 2009;18:2569-78.

29. Dickerman BA, Ahearn TU, Giovannucci E, et al. Weight change, obesity and risk of prostate cancer progression among men with clinically localized prostate cancer. Int J Cancer 2017;141:933-44.

30. Vidal AC, Freedland SJ. Obesity and Prostate Cancer: A Focused Update on Active Surveillance, Race, and Molecular Subtyping. Eur Urol 2017;72:78-83.

31. Pezaro C, Woo HH, Davis ID. Prostate cancer: measuring PSA. Internal Medicine Journal 2014;44:433-40.

32. Umbehr MH, Gurel B, Murtola TJ, et al. Intraprostatic inflammation is positively associated with serum PSA in men with PSA $<4 \mathrm{ng} \mathrm{ml}(-1)$, normal DRE and negative for prostate cancer. Prostate Cancer Prostatic Dis 2015;18:264-9.

33. Boorjian SA, Thompson RH, Siddiqui S, et al. Longterm outcome after radical prostatectomy for patients with lymph node positive prostate cancer in the prostate specific antigen era. J Urol 2007;178:864-70; discussion 870-1.

34. Jayachandran J, Bañez LL, Aronson WJ, et al. Obesity as a predictor of adverse outcome across black and white race: results from the Shared Equal Access Regional Cancer Hospital (SEARCH) Database. Cancer 2009;115:5263-71.

(English Language Editor: A. Kassem) 